\title{
Roswell Park Cancer Institute
}

National Cancer Institute

\section{Source}

National Cancer Institute. Roswell Park Cancer Institute. NCI Thesaurus. Code C39509.

The Roswell Park Institute was founded in 1898 as America's first cancer center. The Institute's goal is to reduce the burden of cancer through close cooperation among basic and translational research, multidisciplinary oncology teams, and educational programs. It was designated as an $\mathrm{NCl}$ comprehensive cancer center in 1974 and is a member of the National Comprehensive Cancer Network. 